read:

$$
D=(1 / 6) \Gamma \hat{\delta}^{2}
$$

As Zener has done, $\Gamma$ may be written as

$$
\Gamma=\nu \exp ^{(\Delta S / R)} \exp (-\Delta H / R T)
$$

where $\nu$ is the characteristic lattice vibration frequency. One then obtains for $D_{0}$ the expression

$$
D_{0}=(1 / 6) \delta^{2} \nu \exp (\Delta S / R)
$$

If the jump distance is assumed to be the mean distance of closest approach, $3.83 \mathrm{~A}$, and the vibration frequency in the liquid is taken to be about the same $\$$ as the Debye

$\$$ Andrade [E. N. da C. Andrade, Proc. Roy. Soc. (London) $215 \mathrm{~A}, 36(1952)]$ has, for example, made similar assumptions in discussing the viscosity of liquids. Evidence that the vibration frequency of many normal metals changes only slightly on fusion frequency for the solid $\left(\theta_{D}=159^{\circ} \mathrm{K}\right)$, we obtain $0.7 \mathrm{eu}$ as the entropy of activation for diffusion in liquid sodium. In view of the uncertainties in jump distance (essentially an admission of our lack of detailed knowledge of the liquid structure) and of the vibration frequency in the liquid, it is possible to say only that the entropy of activation for diffusion in liquid sodium is quite small . . . perhaps zero. 11

is deduced by Kleppa [G. Careri and A. Paoletti, Nuovo cimento $11,399(1954)]$ from measurements of ultrasonic velocities. According to the latter the Grüneisen constant changes from 1.25 for solid sodium to 1.18 for the liquid.

"In this connection it is interesting to note that the activation entropies for self-diffusion in mercury ${ }^{3}$ and in indium ${ }^{14}$ calculated in the same manner are somewhat less than zero if the jump distance is approximated by the interatomic distance. Smaller "jump distances" and positive or zero activation entropies would be possible if diffusion in the liquid were to involve cooperative movements of neighboring atoms.

\title{
Refined Procedure for Analysis of Electron Diffraction Data and Its Application to $\mathrm{CCl}_{4}$
}

L. S. Barteli, ${ }^{*}$ L. O. Brockway, and R. H. Schwendeman

Department of Chemistry, University of Michigan, Amn Arbor, Michigan

(Received July 19, 1954)

\begin{abstract}
$A$ refined procedure for obtaining the structure of free molecules from electron diffraction data is described which compensates for the interference arising from non-nuclear scattering. The procedure is applied to $\mathrm{CCl}_{4}$ using somewhat more extensive rotating sector data than has hitherto been published for this molecule. Estimates are made for the first time in electron diffraction results of the effect of anharmonicity of vibration on the measurement of internuclear distance and of the effect of the failure of the Born approximation on the measurement of amplitudes of vibration. A method of estimating the reliability of the results is described.
\end{abstract}

$\mathrm{R}^{\mathrm{E}}$ ECENT advances in the experimental technique of electron diffraction by gases have made it possible to obtain diffraction data of considerably greater accuracy than that previously available. It is no longer true that the approximations involved in the procedures of analysis in general use are freer from uncertainty than the best experimental data. The availability of punchedcard methods for the calculations, however, has now made it practicable to carry out analyses almost entirely free of uncertainties of interpretation. In addition to yielding more accurate results a more rigorous analysis permits the assignment of uncertainty in a straightforward way from considerations of experimental accuracy.

In electron diffraction it is the scattering of the incident beam by the well-localized nuclei that provides information about the structure of the molecules. The scattering by the diffusely distributed planetary electrons interferes with the measurement of the nuclear scattering at small-to-moderate scattering angles and, unless corrections are made, uncertainties are intro-

\footnotetext{
* Present address: Department of Chemistry, Iowa State College, Ames, Iowa.
}

duced into the determination of the internal motion and the precise location of some of the atoms. A widely used method to compensate partially for electronic scattering is the division of the total scattered intensity curve by the smooth background of atomic scattering. This method, however, fails to take into account the incoherent scattering and the differences between electronic structures of different atoms. A practical procedure for treating this source of error is described in the next section.

If the numerical results are to be significant to within a few thousandths of an angstrom unit, the anharmonicity of the intramolecular vibrations must also be considered. This factor, which has heretofore been ignored in diffraction studies of bonded distances, is responsible for shifting the apparent internuclear distances obtained by conventional procedures of analysis to values of the order of $0.01 \mathrm{~A}$ greater than the true equilibrium internuclear distances. ${ }^{1}$ Another factor neglected in the past is that electrons scattered by atoms undergo a shift in phase which is dependent upon both the a tomic number of the atoms and the angle of scatter-

${ }^{1}$ L. Bartell, J. Chem. Phys. 23, 1219 (1955). 
ing. For atoms only moderately different in a tomic number the influence upon the scattering pattern of molecules is not unlike that of thermal motion, and accordingly, corrections must be made in the determination of amplitudes of vibration between pairs of atoms of different atomic number. ${ }^{2}$ If the difference in atomic number is large the measurement of internuclear distance also will be affected. Compensations are made for the above factors in the results to be presented.

Carbon tetrachloride was selected for this investigation in part because it has been used so frequently in earlier modifications of the electron diffraction method and also because we needed the best possible parameter values in this molecule for comparison with the results in a new study of several substituted chloromethanes. Carbon tetrachloride as a reference molecule has the advantage of a known configuration and of providing a strong interference pattern, but it is not as amenable to independent standardization of its scale factor by spectroscopic means as some other molecules. The refined results are of interest nonetheless because of the many previous investigations and of the convenient opportunity it affords for a full description of the diffraction techniques used in this laboratory.

\section{EFFECT OF NON-NUCLEAR SCATTERING}

In electron diffraction investigations of the structure of free molecules it is customary to work with the ratio, $M(s)$, of the molecular scattering, $I_{M}$, to the atomic scattering, $I_{A}$. If appropriate experimental conditions are satisfied (e.g., single scattering, good localization of the specimen, absence of extraneous scattering, etc.), $M(s)$ assumes a form that can be readily interpreted in terms of molecular structure. In the procedure to follow it is assumed that the ideal experimental conditions have been met or that suitable corrections have been made in the intensity data. An experimental measure of $M(s)$ is obtained from the curve of the total scattered intensity, $I_{T}$, by drawing a smooth background, $I_{B}$, through the molecular oscillations with the aid of criteria $^{3,4}$ to make $I_{B}$ approximate $I_{A}$ closely, so that

$$
M(s)=\left(I_{T} / I_{B}\right)-1 .
$$

The theoretical relationship between $M(s)$ and molecular structure is given by

$$
M(s)=\sum_{i} \sum_{j}{ }^{\prime} C_{i j} \mu_{i j}(s) \int_{0}^{\infty} P_{i j}(r)(\sin s r) / s r d r
$$

${ }^{2}$ V. Schomaker and R. Glauber, Nature 170, 291 (1952); Phys. Rev. 89, 667 (1953). L. Bartell and L. Brockway, Nature 171, 978 (1953).

3 J. Karle and I. L. Karle, J. Chem. Phys. 18, 957 (1950).

4 Bauer, Keidel, Harvey, Coffin, and Bregman, Final Report on project covered by contract N6ori-213, Task Order NR052-040 Cornell University (1950); K. P. Coffin, Doctoral thesis, Cornell University, 1951; S. H. Bauer, ASXRED meeting, Pennsylvania State College, June 1949; S. H. Bauer, Second International Congress of Crystallography, Stockholm, 1951.

5 P. Debye, J. Chem. Phys. 9, 55 (1941).

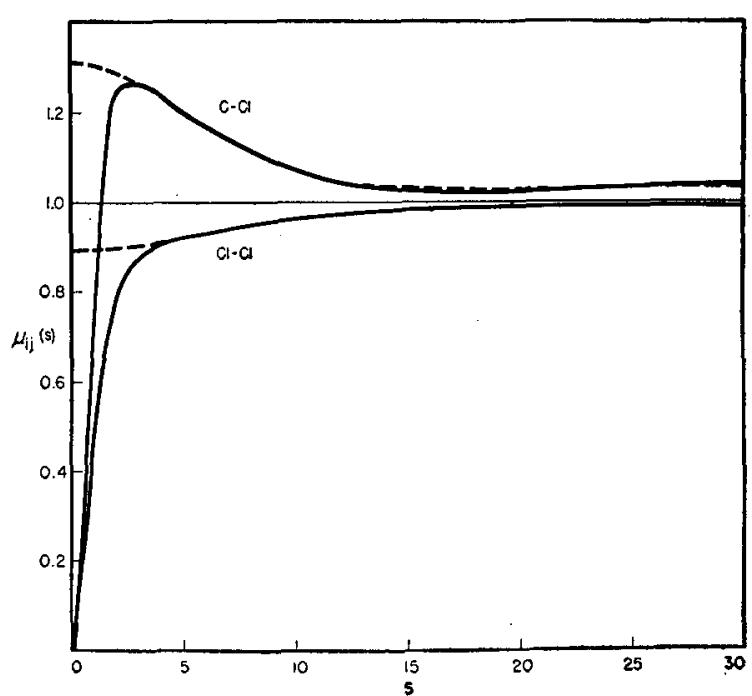

FIG. 1. The functions $\mu_{i j}(s)$ for $\mathrm{CCl}_{4}$. Solid line=theoretical; dashed line $=$ curve-fit representation using Gaussian functions.

where $C_{i j}=Z_{i} Z_{j} / \sum_{i}\left(Z_{i}{ }^{2}+Z_{i}\right), Z_{i}$ is the atomic number of the $i$ th atom in the molecule, $s$ is the variable $\left(4 \pi \sin \frac{1}{2} \theta\right) / \lambda, \theta$ is the scattering angle, $\lambda$ is the wavelength of the electron beam, and $P_{i j}(r)$ is the probability distribution describing the separation, $r$, between nuclei $i$ and $j$. The functions $\mu_{i j}$ are related to the electronic structure of the atoms in the molecule by the following expression :

$$
\mu_{i j}=\frac{\left\{Z_{i}-F_{i}(s)\right\}\left\{Z_{j}-F_{j}(s)\right\} \sum_{i}\left(Z_{i}{ }^{2}+Z_{j}\right)}{Z_{i} Z_{j} \sum_{i}\left\{\left[Z_{1}-F_{i}(s)\right]^{2}+Z_{i} S_{i}(s)\right\}}
$$

in which $F_{i}(s)$ is the x-ray atomic scattering factor and $S_{i}(s)$ is the x-ray incoherent scattering function of atom $i$. If all the internuclear distances are considered to be undergoing harmonic variations, Eq. (2) reduces to

$$
M(s)=\sum_{i} \sum_{j}^{\prime} C_{i j \mu_{i j}} \exp \left(-\ell_{i j}{ }^{2}{ }^{2} / 2\right)\left(\sin s r_{i j}\right) / s r_{i j},
$$

where $\boldsymbol{r}_{i j}$ is the equilibrium separation between nuclei $i$ and $j$ and $\ell_{i j}$ is the root mean square of the amplitude of displacement $\left(r-r_{i j}\right)$.

It is evident from the form of Eq. (2) that information about internuclear spacings in the form of distribution curves, $P_{i j}(\boldsymbol{r})$, could be derived directly from a knowledge of $M(s)$ by Fourier inversion if the functions $\mu_{i j}$ were constant with respect to $s$. An examination of Eq. (3) shows that the $\mu_{i j}$ do approach constancy (unity) at moderate to large $s$ but may deviate by a large amount at small $s$. In general the deviations from unity are larger, the larger the differences that exist between atomic numbers in the molecule. Figure 1 shows the functions for $\mathrm{CCl}_{4}$.

The Norwegian school takes into account the variability of $\mu_{i j}$ with $s$ but in a way that makes the interpretation of the distance between the weaker scattering centers in molecules with several similar distances 
rather tedious. ${ }^{6}$ Most of the other investigators have neglected the variability of $\mu_{i j}$ except at very small $s$ and have reduced diffraction data to molecular structure by two methods ${ }^{7}$ : (a) the correlation method in which experimental $M(s)$ curves are compared to constant coefficient theoretical curves, $M_{c}(s)$, calculated for proposed molecular models by means of Eq. (2) or Eq. (4) with the assumption that $\mu_{i j}=1$, and (b) the radial distribtion method in which an approximate Fourier analysis is performed upon the experimental intensity function to obtain a radial distribution curve, $f(\boldsymbol{r})$, where

$$
f(r)=\int_{0}^{s(\max )} s M(s) \exp \left(-b s^{2}\right) \sin s r d s .
$$

In the correlation method the great disparity between $M(s)$ and $M_{c}(s)$ at very small $s$ is not serious because the selection of the most likely model can be based on comparison outside that range. In the radial distribution method, however, the value of the integrand of Eq. (5) from $s=0$ to some small value is important in determining the form (but not the position of the essential features) of the radial distribution curve $f(r)$. Consequently, the interpretation of the radial distribution curve is facilitated by substituting for the experimental $M(s)$ a calculated constant coefficient curve in the range from $s=0$ to $s \sim 3$. The resulting $f(r)$ curve is closely related to the $P_{i j}(r)$ functions and can be used to obtain a measure of both the internuclear distances, $r_{i j}$, and the amplitudes of vibration, $\ell_{i j}{ }^{8}$

The deviation of the $\mu_{i j}$ from constancy in the range from $s=3$ to $s=20$ is less pronounced than the deviation at smaller $s$, but it is nevertheless sufficient that its neglect reduces the applicability of the criteria used to establish the background line, $I_{B}$, and makes uncertain the measurements of the "index of resolution" of the pattern $\left\{M_{\exp }(s) / M_{\text {theor }}(s)\right\}$, the amplitudes of vibration, the distances between the weaker scatterers in a molecule, and the actual resolution of approximately equal internuclear distances. The most serious obstacle to the more exact treatment has heretofore been the excessive labor involved in the computation. It is shown below how the corrections can be carried out by a simple extension of the punched-card computational procedure described by Karle and Karle. ${ }^{3}$

The compensation for variable $\mu_{i j}$ is applied here by converting the experimental $M(s)$ function to a function with constant coefficients, $M_{c}(s)$, which is then used in computing the distribution function and in correlation with the theoretical $M(s)$ functions calculated from Eq. (4) with $\mu_{i j}=1$. This conversion uses a difference

${ }^{6}$ H. Viervoll, Acta Chem. Scand. 1, 120 (1947).

${ }^{7} \mathrm{~S}$. H. Bauer et al. have proposed a method for partially compensating for the variability; see $K$. P. Coffin (Doctoral thesis, Cornell University, 1951). Schomaker has often pointed out the effect of the $\mu_{i j}$ on the radial distribution curve. Other investigators have occasionally made compensations in the correlation method.

${ }^{8}$ I. L. Karle and J. Karle, J. Chem. Phys. 17, 1052 (1949). function, $\Delta M$, given by

$\Delta M=M(s)-M_{c}(s)$

$$
\begin{aligned}
=\sum_{i} \sum_{j}^{\prime} c_{i j}\left(\mu_{i j}-1\right) & \times \exp \left(-\ell_{i j}{ }^{2} s^{2} / 2\right)\left(\sin s r_{i j}\right) / s r_{i j},
\end{aligned}
$$

in which the $\mu_{i j}$ can be computed from tabulated values of $F_{i}(s)$ and $S_{i}(s)$. The correction, $\Delta M$, is not usually very sensitive to assumptions about the structure of the molecule because $\left(\mu_{i j}-1\right)$ damps out rapidly unless large differences exist between atomic numbers. In any case, it is possible to arrive at $\Delta M$ by successive approximations, starting with values of $r_{i j}$ and $\ell_{i j}$ obtained from an uncorrected radial distribution curve. If $\left(\mu_{i j}-1\right)$ can be expressed in terms of a series of Gaussian functions such that

$$
\left(\mu_{i j}-1\right)=\left\{\sum_{n} G_{n} \exp \left(-g_{n} s^{2}\right)\right\}_{i j},
$$

then the function $\Delta M$ can be computed by exactly the procedure prescribed for the theoretical $M(s)$ in Eq. (4) with $\mu_{i j}=1$. The utility of this method lies in the fact that $\Delta M$ can be calculated readily by punched-card methods and that a good curve fit of $\left(\mu_{i j}-1\right)$ can be obtained with a small number of terms. It has been our experience that, for molecules containing atoms no heavier than $\mathrm{Cl}$, the $\left(\mu_{i j}-1\right)$ function can often be fitted in the range from $s=3$ to $s_{\max }$ by a small constant, $G_{0}$, and one term with $g_{n} \neq 0$. It is seldom necessary to use more than two terms with $g_{n} \neq 0$ for light molecules.

The range of intensity data from $s=0$ to $s=3$, which cannot be treated satisfactorily in this manner, is insensitive to molecular structure parameters and hence can be ignored completely in the correlation method. Compensation for this range in the radial distribution method can be made as already described. The corrected experimental curve, $M_{c}(s)=M(s)-\Delta M$, can then be analyzed in terms of the spatial distribution of nuclei in the molecule, the corrections to be described for the failure of the Born approximation and anharmonicity of intramolecular vibrations being most conveniently made after the correlation or radial distribution treatment has been performed.

The primary advantage of the foregoing treatment over previous treatments designed to take into account the effect of non-nuclear scattering is that the radial distribution function can be interpreted as the sum of a number of simple bell-shaped peaks. ${ }^{9}$ Each internuclear distance gives rise to just one such peak instead of several regions of positive and of negative amplitudes as is the case in the Norwegian treatment. Further, the area of each component peak as measured directly from the base line of $f(r)$ is simply related to the scattering powers of the atoms involved, and the breadth of each

\footnotetext{
${ }^{9}$ It should be noted that the treatment described here has been developed for molecules whose distances show harmonic variations [see Eqs. (2) and (4)], but the application to molecules having nonharmonic motions, such as internal rotations, is readily made. In either case the base line to which the resolved peaks are properly referred is flat and should lead to more precise peak areas.
} 
ANALYSIS OF ELECTRON

component is readily interpreted in terms of amplitude of vibration without further consideration of effects of the $\mu_{i j}$. The greater ease of resolving a complex peak into its components by means of this scheme is apparent. It is only fair to note that the rapidity with which the scheme converges, if very heavy atoms are present, has not been tested quantitatively.

\section{EXPERIMENTAL PROCEDURE}

The electron diffraction apparatus used in this research and the technique used to obtain the diffraction patterns are described in detail elsewhere. ${ }^{10}$ Patterns were taken of $\mathrm{CCl}_{4}$ vapor at $20 \mathrm{~mm}$ pressure with an $R^{3}$ sector, using specimen-to-plate distances of 25 and 10 $\mathrm{cm}$ to record the inner and outer portion of the patterns. The diffraction data, which included the first fifteen interference maxima, extended to $s=34$ at which point the internal motion of the molecule had reduced the amplitude of the sinusoidal molecular interference terms to about $7 \%$ of the amplitude calculated for a rigid molecule. Microphotometer traces were obtained by the method described by Karle, Hoober, and Karle ${ }^{11}$ in which patterns are spun about their centers while being scanned in order to average out irregularities in the photographic emulsion. Optical densities were obtained by reading the amplified microphotometer records under 10 power magnification with a device for accurate interpolation, and these readings were converted to relative intensities. $^{12}$

A background line which agreed well with the theoretical atomic intensity curve was drawn through the experimental intensity curve with the aid of suitable criteria $^{3,4}$ in order to separate the contributions of the atomic and molecular scattering. The experimental $M(s)$ curve was computed according to Eq. (1) and corrected to a constant coefficient curve according to Eq. (6), using for the $\mu_{i j}$ the approximations

$$
\begin{gathered}
\mu_{\mathrm{CCl}}-1=0.030+0.278 \exp \left(-0.0189 s^{2}\right), \\
\mu_{\mathrm{ClCl}}-1=-0.009-0.0951 \exp \left(-0.0124 s^{2}\right),
\end{gathered}
$$

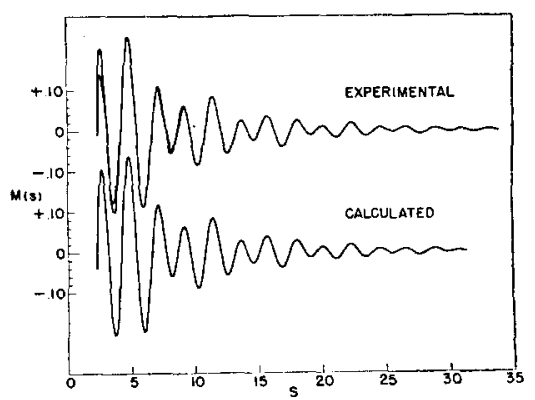

FIG. 2. Comparison of experimental and calculated $M(s)$ curves for $\mathrm{CCl}_{4}$. In the experimental curve the dashed line represents the uncorrected $M(s)$, and the solid line represents the corrected constant coefficient $M_{c}(s)$. The lower curve is the theoretical constant coefficient $M_{c}(s)$ using in the exponential factor the experimental $\ell$ 's (Table I).

${ }^{10}$ L. Brockway and L. Bartell, Rev. Sci. Instr. 25, 569 (1954).

${ }^{11}$ Karle, Hoober, and Karle, J. Chem. Phys. 15, 765 (1947).

${ }^{12}$ L. Bartell and L. Brockway, J. Appl. Phys. 24, 656 (1953).
DIFFRACTION DATA, $\mathrm{CCl}_{4}$

1857

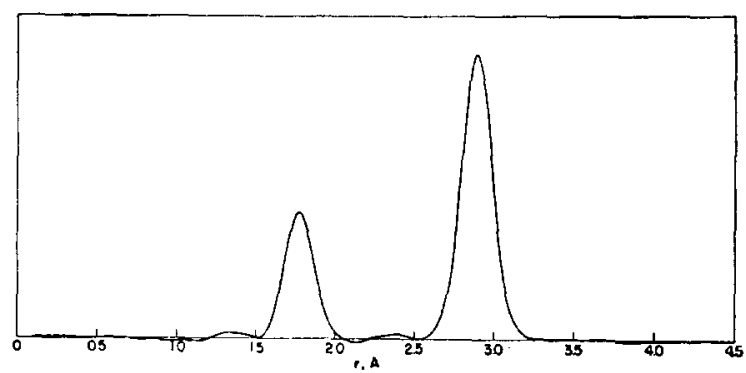

FIG. 3. Experimental radial distribution curve for $\mathrm{CCl}_{4}$.

which can be compared with the theoretical $\mu_{i j}$ in Fig. 1. Figure 2 compares the uncorrected and corrected experimental $M(s)$ curves with the theoretical constant coefficient curve.

The radial distribution function for $\mathrm{CCl}_{4}$ shown in Fig. 3 was calculated with the corrected data according to Eq. (5) by the punched-card method described by Shaffer, Schomaker, and Pauling, ${ }^{13}$ using a value of 0.0023 for the constant $b$. A calculation showed that this value of $b$ was adequate to reduce to a negligible amount the extraneous ripple arising from the cutoff of the integral at $s_{\max }$, since the intramolecular motion of the molecule itself contributed strongly to damping the integrand. At the same time, $b$ is not so large that the effective weight function, $s \exp \left(-b s^{2}\right)$, imposed upon the diffraction data places an unduly small emphasis on the outer portion of the data.

\section{UNCERTAINTIES}

The limits of error usually assigned to electron diffraction results in the past were relatively large compared to the apparent internal consistency of the experimental data. This largeness was dictated by experience with many determinations instead of by straightforward analysis in any given case. It seems reasonable to ascribe a large part of the need for this empirical factor of caution to failures in the approximations used in the analysis and interpretation of data. Since more rigorous methods were used in this investigation, the estimation of the reliability of the results presented here was based upon an examination of the uncertainty in the experimental data and the uncertainty in the corrections for anharmonicity.

Experimental uncertainties were separated into three categories, the first of which included errors in the settings and calibration of the diffraction apparatus and systematic errors in the measurement of the scattering angle. Such errors primarily affect the determination of the scale factor of internuclear distances rather than molecular shape. Checks of the apparatus, the uniformity in thickness and in flatness of the photographic plates, and the tracing by the microphotometer indicated that the uncertainty due to this category

${ }^{13}$ Shaffer, Schomaker, and Pauling, J. Chem. Phys. 14, 659 (1946). 
amounts to about one part per thousand in the present investigation.

A second category included random errors associated with the measurement of intensity as a function of scattering angle. These errors, which affect the determination of the shape of molecules and amplitude of vibrations as well as the size, reveal themselves in the correlation method as a scattering of the $s / s_{0}$ values and in the radial distribution curve as random fluctuations appearing in the background between the peaks. This latter evidence was used to estimate uncertainties of the second category in $r_{i j}$ and $\ell_{i j}$ by determining the extent to which fluctuations in the distribution curve of the observed amplitude and breadth were capable of altering the positions and breadths of the peaks. The average breadth of the fluctuations observed in curves obtained by the present method is comparable to the breadths of the internuclear peaks. It can be shown that if the amplitude of the fluctuations is $y$ in the vicinity of a peak of height $A$, then the uncertainty in $\ell_{i j}$ and $r_{i j}$ is given very roughly by

$$
\delta r_{i j} \sim \delta \ell_{i j} \sim y\left(2 b+\ell_{i j}^{2}\right)^{\frac{1}{2}} / A .
$$

A more precise evaluation of the uncertainty requires a closer examination of the form of the disturbance. The value of $y$ observed in our current investigations has generally been one or two percent of the height of the strongest peaks.

The third group of uncertainties included the systematic errors in intensity measurement, such as in the determination of the slope of the calibration curve relating optical density to intensity and in the failure to meet ideal diffraction conditions. The absence of serious extraneous scattering was inferred from the agreement of the theoretical and experimental background curves, the high index of resolution of the pattern at small $s(\sim 93 \%)$ and tests of the distribution of the gas sample in the diffraction chamber ${ }^{10}$ These errors, which mainly affect the extent of damping of $M(s)$, are not adequately represented by the random fluctuations in the distribution function because they influence the shape of the peaks more than the base line between the peaks. The distortion, being more or less symmetrical about each peak, has a significant effect only on the measurement of the $\ell_{i j}$ unless the systematic errors are quite large. The associated uncertainty in $\ell_{i j}$ in the present investi-

TABLE I. Distances and amplitudes in $\mathrm{CCl}_{4}$.

\begin{tabular}{lccc}
\hline & Internuclear distance (A) & $\ell_{i i^{\prime} \text { (elec. diff) }}$ & $\ell_{i j^{\prime} \text { (spect.) }}$ \\
\hline $\mathrm{C}-\mathrm{Cl}$ & $\begin{array}{r}r_{R D}=1.769 \pm 0.005^{\mathrm{a}} \\
r_{R D^{0}}=1.766 \pm 0.003^{\mathrm{b}} \\
r_{t}=1.760 \pm 0.004^{\mathrm{a}}\end{array}$ & $0.060 \pm 0.005$ & 0.054 \\
$\mathrm{Cl}-\mathrm{Cl}$ & $r_{R D}=2.887 \pm 0.004^{\mathrm{a}}$ & $0.068 \pm 0.003$ & 0.067 \\
\hline
\end{tabular}

\footnotetext{
$=r_{R D}=$ position of maximum of symmetrical curve best fitting upper part of experimental $R D$ curve.

b $r_{R D^{0}}=$ position of maximum of $P_{i j}(r) / r$ curve.

- $r_{\theta}=$ equilibrium separation.
}

gation was estimated to be $0.002 \mathrm{~A}$ by considering the envelope of the damping of the molecular terms of $M(s)$ in relation to the estimated uncertainties of $3 \%$ [of the amplitude of $M(s)]$ in the index of resolution at small values of $s$ and of $1.5 \%$ of $M(s)$ in the emulsion calibration curve. In addition the uncertainity owing to the measured spread of the specimen was estimated and found to be less important than the preceding factors.

\section{RESULTS}

\section{Amplitudes of Vibration}

It was shown by Karle and Karle ${ }^{8}$ that the amplitudes of intramolecular motion, the $\ell_{i j}$ of Eq. (4), can be studied by refined electron diffraction methods. It has recently been pointed out, ${ }^{2}$ however, that the apparent amplitudes, $\ell_{i j}$, obtained by the application of Eq. (4) or Eq. (5), must be corrected for the failure of the Born approximation upon which Eqs. (4) and (5) are based. The correction for $40 \mathrm{kv}$ electrons is given approximately by

$$
\ell_{i j}{ }^{\prime}=\left\{\ell_{i j}{ }^{2}-3.2 \times 10^{-6}\left(Z_{i}-Z_{j}\right)^{2}\right\}^{\frac{1}{2}} .
$$

The difference between $\ell_{\mathrm{CCl}}{ }^{\prime}$ and $\ell_{\mathrm{CCl}}$ is $0.003 \mathrm{~A}$ for $\ell_{\mathrm{CCl}}=0.060 \mathrm{~A}$ and is $0.004 \mathrm{~A}$ for $\ell_{\mathrm{CCl}}=0.040 \mathrm{~A}$. The small asymmetry of the peaks does not interfere with the measurement of their breadth.

The experimental results, obtained from the radial distribution curve, are listed in Table $I$ and are in satisfactory agreement with the spectroscopic results as calculated by Morino ${ }^{14}$ et al. but higher than the only other electron diffraction values ${ }^{8}\left(\ell_{\mathrm{CCl}}=0.041\right.$ $\left.\pm 0.005 \mathrm{~A} ; \ell_{\mathrm{ClCl}}=0.054 \pm 0.005 \mathrm{~A}\right)$.

\section{Internuclear Distances}

It is of interest to report the values of the internuclear distances in $\mathrm{CCl}_{4}$ obtained from the diffraction data with the assumption that the radial distribution peaks are symmetrical with respect to the equilibrium distances, for it is these values which should be compared to previous published results of electron diffraction investigations. Fitting the upper two-thirds of the radial distribution peaks with symmetrical Gaussian functions gave values, $r_{R D}$, of $1.769 \pm 0.005 \mathrm{~A}$ for the $\mathrm{C}-\mathrm{Cl}$ peak and $2.887 \pm 0.004 \mathrm{~A}$ for the $\mathrm{Cl}-\mathrm{Cl}$ peak, the uncertainties representing all of the known sources of experimental error without any consideration of errors of interpretation. Since the $\mathrm{C}-\mathrm{Cl}$ peak is less precisely determinable from the diffraction data than the stronger $\mathrm{Cl}-\mathrm{Cl}$ peak a somewhat more precise value of the equilibrium $\mathrm{C}-\mathrm{Cl}$ distance can be found from the latter peak, assuming tetrahedral symmetry, than from the $\mathrm{C}-\mathrm{Cl}$ peak directly. It should be noted, however, that intramolecular vibrations complicate the computation, and, in general, it is not to be expected that the positions

${ }^{14}$ Morino, Kuchitsu, Takahashi, and Maeda, J. Chem. Phys. 21, 1927 (1953). 
of the peak maxima will be exactly in the ratio calculated for a rigid tetrahedral molecule. Application of the correlation procedure leads to the same internuclear distance as the radial distribution method to within $0.001 \mathrm{~A}$. These results are in agreement with all but the oldest values in the literature to within the reported uncertainties.

The foregoing results are not to be interpreted as true equilibrium distances (in the spectroscopic sense) because of the distortions introduced by the anharmonicities of the intramolecular vibrations. In order to estimate the effect of anharmonicity it was assumed that the interaction between the bonded atoms could be represented by a Morse function

$$
V(r)=D\left\{e^{-2 a\left(r-r_{e}\right)}-2 e^{-a\left(r-r_{\bullet}\right)}\right\},
$$

where $r_{e}$ is the equilibrium distance, $D$ is a constant equal to the dissociation energy, and $a=(k / 2 D)^{\frac{1}{2}}$ in which $k$ is the force constant. The probability distribution, and hence, the radial distribution peak, of the $\mathrm{C}-\mathrm{Cl}$ distance is then characterized by three molecular parameters, $r_{e}, \ell_{i j}$, and $a$, all of which could be determined, in principle, from accurate data. In practice the data are not sufficiently precise to fix the anharmonicity parameter $a$ but a reasonable value for $a$ can be easily estimated from spectroscopic or thermal measurements involving $\mathrm{C}-\mathrm{Cl}$ bonds. The values for $r_{e}, \ell_{i j}$, and a new parameter $r_{R D}{ }^{0}$, the position of the maximum of the nuclear radial distribution curve $P_{i j}(r) / r$, (usually $\left.r_{R D}>r_{R D}{ }^{0}>r_{e}\right)$ are readily found by fitting the experimental peaks with Morse-type distribution functions once a value for $a$ is assumed. ${ }^{1}$ It was assumed that $a=2.0 \mathrm{~A}^{-1}$ for the $\mathrm{C}-\mathrm{Cl}$ bond. The theoretical treatment of the asymmetry of the $\mathrm{Cl}-\mathrm{Cl}$ peak is an exceedingly difficult problem and is beyond the scope of the present investigation. It seems reasonable, however, to assume that the distribution describing the $\mathrm{Cl}-\mathrm{Cl}$ separation can be represented approximately by a Morse-type distribution function with a value of $a$ between 2 and 4. Fortunately, the result for the weighted $\mathrm{C}-\mathrm{Cl}$ distance is not unduly sensitive to the assumptions made about the $\mathrm{Cl}-\mathrm{Cl}$ distribution. The different distance parameters listed in Table I for the $\mathrm{C}-\mathrm{Cl}$ bond were arrived at on the above basis.

It would be expected that $r_{R D}$ would converge to $r_{R D}{ }^{0}$ with decreasing $b$ and sufficiently large $s_{\max }$. For the $\mathrm{C}-\mathrm{Cl}$ bond in $\mathrm{CCl}_{4}, r_{R D^{0}}$ is very nearly the same at $0^{\circ}$ as at $300^{\circ} \mathrm{K}$ because of the cancellation of the small effects of vibration and rotation. Perhaps more significant parameters for describing the internuclear distance are the position of the maximum of the best fit $P_{\mathrm{C}-\mathrm{Cl}}(r)$ which is at $1.768 \mathrm{~A}$, or the center of gravity which is at about $1.771 \mathrm{~A}$.

It is reasonable to inquire whether the experimental intensity curve itself showed any evidence of anharmonicity or need for the foregoing corrections. The answer is that the effects sought are so small that they

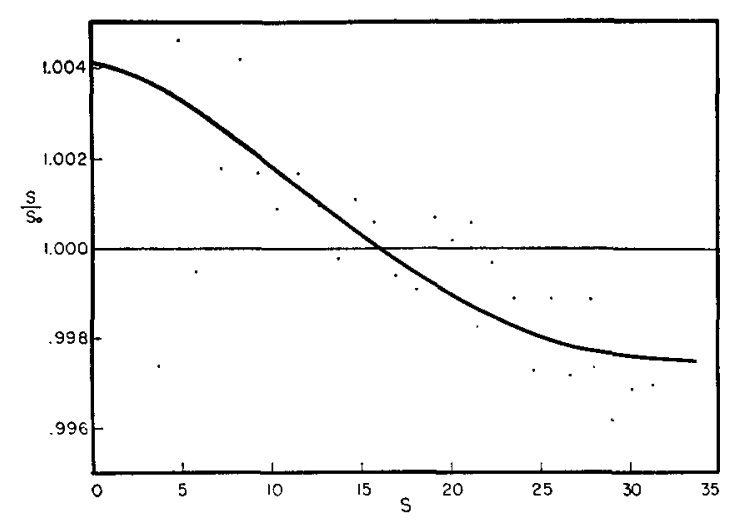

FIG. 4. Curve $=$ the dependence of the $s / s_{0}$ values upon $s$ that is estimated to hold for $\mathrm{CCl}_{4}$ at $300^{\circ} \mathrm{K}$; the deviation of this curve from constancy is a measure of the anharmonicity of intramolecular vibrations. Points $=$ experimental $s / s_{0}$ correlation values for $\mathrm{CCl}_{4}$.

approach in magnitude the experimental uncertainties but that the diffraction data are somewhat better represented by the anharmonic model than the harmonic model. Anharmonicity manifests itself in the radial distribution method by introducing slight asymmetry into the peaks and in the correlation method by imparting a more or less smooth frequency modulation into the $M_{\text {exp }}(s)$ curve. It has been noted in most of the radial distribution curves obtained in this laboratory by the careful application of the rotating sector method that the peaks do show slight asymmetry of the expected magnitude and direction. The peaks for $\mathrm{CCl}_{4}$ shown in Fig. 3 possess this characteristic although it is apparent that the random fluctuations in the base line interfere with the evaluation of the effect. Figure 4 compares the experimental $s / s_{0}$ values with the values estimated to occur for $\mathrm{CCl}_{4}$ vibrating asymmetrically at $300^{\circ} \mathrm{K}$. For the outer $22 s / s_{0}$ values the mean deviation from the anharmonic curve is about 8 parts per 10000 , while the mean deviation from constancy (harmonic vibrations) is about 15 parts per 10000 .

The above distinctions between the equilibrium internuclear distance and other naturally derived distance parameters were made in the present investigation because it was believed that such distinctions were necessary to keep the error in interpretation as small as the experimental error.

\section{ACKNOWLEDGMENTS}

We appreciate the support of the Horace H. Rackham School of Graduate Studies in providing a Rackham Post-Doctoral Fellowship for L. S. Bartell and the Henry Earle Riggs Fellowship for R. H. Schwendeman. We are very grateful to Dr. J. Karle and the Naval Research Laboratory for a deck of cards punched with exponential functions and to Dr. V. Schomaker and the California Institute of Technology for cards punched with sine functions. 